\title{
Spontaneous Four Limb Compartment Syndrome
}

\author{
Giovanna Riolo MD, Danny Arora MD, David Taylor MD, Gavin CA Wood MBChB, FRCS
}

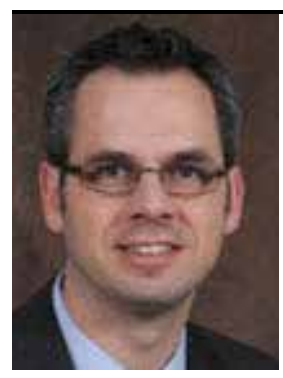

Taylor

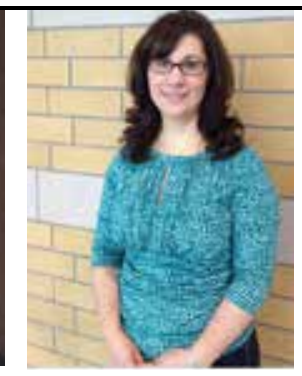

Riolo

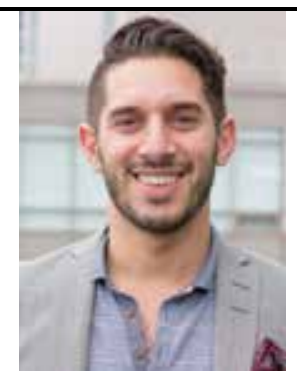

Arora

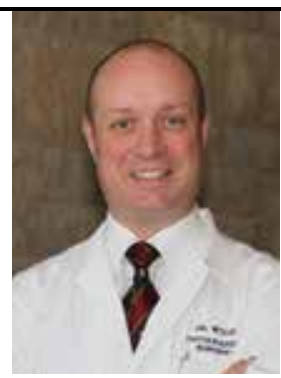

Wood

\section{About the Authors}

David Taylor, $M D$ is a general internist at Queen's University. Giovanna Riolo MD, FRCPC is a respirologist and cystic fibrosis specialist at University of Toronto, Toronto. Danny Arora MD, MSc (c), FRCSC - Orthopaedic Surgery Clinical Fellow, University of Toronto. Gavin Wood MBChB, FRCS is an orthopedic surgeon at Queen's University. Correspondence may be directed to riologi@smh.ca.

\section{Summary}

We present a unique case of atraumatic rhabdomyolysis and four-limb compartment syndrome. Edema and inflammation associated with rhabdomyolysis, was further exacerbated by aggressive saline resuscitation. This resulted in raised compartment pressures and necessitated four-limb fasciotomy. The cause of rhabdomyolysis was most likely multifactorial including systemic capillary leak syndrome seizure, illicit drug use (crack cocaine and methamphetamine) and the prescription medication, mirtazapine.

\section{Résumé}

Nous présentons ici un cas exceptionnel de rhabdomyolyse atraumatique accompagnée d'un syndrome des loges aux quatre membres. L'œdème et l'inflammation associés à la rhabdomyolyse ayant été exacerbés par une réanimation liquidienne agressive, il en est résulté une augmentation de la pression dans les loges et il a fallu procéder à une aponévrotomie aux quatre membres. La rhabdomyolyse a été très probablement causée par plusieurs facteurs, dont une crise systémique d'un syndrome de fuite capillaire et l'usage illicite de drogues (cocaïne épurée ou crack, méthamphétamine) jumelé au médicament prescrit, la mirtazapine. 


\section{Case}

A 49-year-old female presented to a community hospital emergency department after awaking with bilateral hand and forearm swelling and diffuse myalgias that restricted motion. The myalgias were not limited to pressure areas. She denied recent exercise or trauma. Although an epileptic, she denied recent seizures. She admitted to smoking crack cocaine and marijuana daily and injecting crystal methamphetamine five days prior.

Past history included hepatitis B, chronic hepatitis C and (untreated) epilepsy. She took Mirtazepine for depression. For 5 months, she had taken Isoniazide, Rifampin and Pyridoxine for pulmonary tuberculosis therapy. Five months prior, she consumed approximately 6 beers per day, twice weekly.

On examination, she was normothermic, pulse was 110 beats per minute and blood pressure 150/90 mmHg. Cardiac, pulmonary, abdominal, and neurologic exams were unremarkable. She had bilateral edema of hands and forearms but preserved distal pulses, and slightly limited motion of wrists and metacarpal phalangeal joints. Urine output was $50 \mathrm{cc} / \mathrm{hr}$.

She had an elevated creatinine kinase (CK) at 23,480 U/L (normal (N): 35-155), aspartate aminotransferase at $3211 \mathrm{U} / \mathrm{L}$ (N: 5-40) and alanine aminotransferase at $543 \mathrm{U} / \mathrm{L}$ (N: 0-35). Complete blood count, alkaline phosphatase, total bilirubin, troponin, renal function, lactate, blood cultures and thyroidstimulating hormone were normal. Myoglobin was detected in the urine. A urine toxicology screen was positive for opiates and cannabinoids.

Work-up included serum HIV, Epstein-Barr virus and cytomegalovirus testing, which were negative. Given elevated liver enzymes, HCV RNA viral load was sent, but was undetectable along with a normal abdominal ultrasound. Anti-nuclear antibody showed a positive homogenous pattern with a low titer of 1:160 and smooth muscle antibodies were negative.

A working diagnosis of atraumatic rhabdomyolysis and myositis was assumed. During hospitalization, she had episodes of hypovolemia and low jugular venous pressure. In response to these hemodynamic changes, a total of seven liters of $0.9 \%$ normal saline was infused over 18 hours from admission. The following morning after admission, her upperextremity myalgia was worse, as was ambulation. All limbs were very edematous, and tender to palpation and movement. Urine was now tea-coloured; though output was still adequate (70cc/hr). CK-total peaked at 186,913 U/L.

Concern of acute compartment syndrome led to urgent transfer for surgical consultation. Compartment pressures, using a transducer, were as follows: right forearm $68 \mathrm{mmHg}$ on dorsal side and $58 \mathrm{mmHg}$ on volar side; her anterior lowerleg pressures measured $40 \mathrm{mmHg}$ on the left and $48 \mathrm{mmHg}$ on the right.

She underwent urgent four-limb fasciotomy: bilateral forearms (volar and dorsal), bilateral carpal tunnel releases, bilateral legs (anterior, lateral, superficial and deep posterior) with irrigation and debridement. Intraoperatively, there were signs of poor muscle perfusion (delayed contraction and palor), but no necrosis. This resolved with surgical release. The extreme swelling meant that full skin closure could not be achieved until day 4 . Muscle biopsy did not identify an etiology for the rhabdomyolysis.

Post-operatively, CK normalized within a week. She was able to have normal mobility in 4 limbs. She had mild paresthesia in the median nerve distribution of the left hand and preserved function of the radial and ulnar distribution.

\section{Discussion}

This case describes atraumatic rhabdomyolysis complicated by four-limb compartment syndrome, and associated most likely with aggressive hydration. Rhabdomyolysis can cause (and be further worsened by) compartment syndrome. ${ }^{1}$ However, atraumatic rhabdomyolysis complicated by four-limb compartment syndrome has not been previously reported. Ninety-seven percent of cases are associated with either a fracture $(69 \%)$ or a traumatic soft-tissue injury (28\%). This leaves a small minority from spontaneous or atraumatic causes. ${ }^{2}$ The cause of our patient's rhabdomyolysis was likely multifactorial, such as recreational drugs (cocaine and methamphetamine), prescription drugs (Mirtazepine and Isoniazide) and/or due to a rare syndrome called systemic capillary leak syndrome.

Cocaine-induced ${ }^{3}$ and alcohol-induced rhabdomyolysis ${ }^{4}$ have been widely reported. Like cocaine, methamphetamine $e^{5,6}$ can cause violent behavior and excessive isometric motor movement. This along with increased adenotriphosphate (ATP) demand can cause rhabdomyolysis. ${ }^{5}$ Methamphetamine users also under hydrate and therefore present with a higher mean initial CK than non-methamphetamine patients. ${ }^{5}$ Although our patient denied alcohol use, alcohol withdrawal resulting in delirium tremens, can also elevate CK. ${ }^{4}$

Prescription drugs such as isoniazid and mirtazapine may have contributed. Isoniazid has been associated with rhabdomyolysis, ${ }^{7}$ but is unlikely in our case given that it had been tolerated for 5 months prior without complications. Our patient was taking Mirtazapine. Mirtazipine lowers seizure-threshold- especially when combined with alcohol- 
withdrawal and benzodiazepines- and seizures can result in rhabdomyolysis. Concurrent use with inhibitors or inducers of CYP1A2, CYP2D6, or CYP3A4 may result in higher or lower concentrations of mirtazapine, respectively.,

Systemic capillary leak syndrome (SCLS) is a severe and rare disease caused by increased capillary permeability. The cause is unknown and characterized by episodes of hypotension, edema and hypovolemia. This results in a massive shift of fluid into the extravascular space and compartment syndrome. ${ }^{10}$ Less than 100 cases have been described since 1960, with only one case of four-limb compartment syndrome. ${ }^{10}$ Intravenous fluids increase the risk of vascular overload without providing an increase arterial pressure. ${ }^{10}$

In our case, in addition to 7 liters of crystalloid fluid in less than 24 hours, intramuscular inflammation, such as occurs with rhabdomyolysis, can mean even more interstitial edema. ${ }^{2,11}$ This along with possible SCLS, plus recreational and prescription drugs, could have worsened the edema. This in turn makes compartment syndrome more likely through further swelling, ischemia and fluid sequestration. All of this can cause a vicious cycle of ongoing muscle damage, and further CK elevation. Continuous monitoring of intracompartmental pressure can help to help reduce complications (including the threat of limb-loss). Surgical fasciotomy was performed to break this medical vicious cycle.

\section{References}

1. Khan FY. Rhabdomyolysis: a review of the literature. Neth J Med. 2009 Oct;67:272-83.

2. McQueen MM, Gaston P, Court-Brown CM. Acute compartment syndrome, Who is at Risk? J Bone Joint Surg Br. 2000 Mar; 82(2):200-3.

3. Horowitz BZ, Panacek EA, Jourites, NJ. Severe rhabdomyolysis with renal failure after intranasal cocaine use. J Emerg Med. 1997 Nov-Dec;15(6):833-7.

4. Curry SC, Chang D, Connor D. Drug- and Toxin-Induced Rhabdomyolysis. Ann Emerg Med. 1989 Oct;18(10):1068-84.

5. Richards JR, Johnson EB, Stark RW, Derlet RW. Methamphetamine Abuse and Rhabdomyolysis in the ED: 5 year study. Am J Emerg Med. 1999 Nov;17(7):681-5.

6. Knochel JP. Mechanisms of rhabdomyolysis. Curr Opin Rheumatol. 1993 Nov;5(6):725-31.

7. Cronkright PJ, Szymaniak G. Isoniazid and Rhabdomyolysis. Annals of Internal Medicine. 1989 Jun 1;110(11):945.

8. Khandat AB, Nurnberger JI Jr, Shekhar A. Possible mirtazapine-induced rhabdomyolysis. Ann Pharmacother. 2004 Jul-Aug;38(7-8):1321.

9. Kuliwaba A. Non-lethal mirtazapine overdose with rhabdomyolysis. Aust N Z J Psychiatry. 2005 April;39(4):312-3.

10. Simon DA, Taylor TL, Bayley G, Lalonde KA. Four-limb compartment syndrome associated with the systemic capillary leak syndrome. J Bone Joint Surg Br. 2010 Dec;92(12):1700-2.

11. Poels PJE, Gabreels FJM. Rhabdomyolysis: a review of the literature. Clin Neurol Neurosurg. 1993 Sep;95(3):175-92. 